\title{
Retrasplante cardíaco: reporte de un caso.
}

\author{
Ernesto Aranguiz-Santander ${ }^{1,2,3}$; Oneglio Pedemonte ${ }^{1,2,3}$; Andrés Vera ${ }^{1,2,3}$; Manuel Quiroz ${ }^{2,3}$; Javier Alburquerque ${ }^{1,3}$; Lorenzo Merello. ${ }^{1,2,4}$ \\ 1. Cirugía Cardiovascular, Hospital Dr. Gustavo Fricke, Viña del Mar, Chile. \\ 2. Cirugía Cardiovascular, Universidad de Valparaíso, Viña del Mar, Chile. \\ 3. Fundación Cardiovascular Dr. Jorge Kaplan Mayer, Viña del Mar, Chile. \\ 4. Anestesia y reanimación, Universidad de Valparaíso, Viña del Mar, Chile.
}

Resumen: El Trasplante cardíaco es la mejor alternativa para la insuficiencia cardíaca terminal, logrando buenos resultados de sobrevida y calidad de vida a largo plazo.

Una de las causas más importantes de morbimortalidad es la falla del injerto, la que puede ser secundaria, entre otros, a rechazo agudo y/o vasculopatía y su presencia requiere considerar todas las alternativas terapéuticas, dentro de las cuales está el retrasplante. Los resultados de sobrevida en retrasplante cardíaco son buenos. No obstante, los pacientes presentan los riesgos de una terapia inmunosupresora más intensa, así como el desarrollo recurrente de vasculopatía del injerto. Por lo que se considera una opción en pacientes cuidadosamente seleccionados, dado que la experiencia internacional demuestra que la sobrevida del retrasplante es menor que en el primer trasplante.

Presentamos el caso de un paciente trasplantado a los 42 años, quien desarrolla una enfermedad vascular del injerto e insuficiencia cardíaca con capacidad funcional IV, por lo cual se decidió realizar un retrasplante cardíaco.

Palabras Clave: Falla del aloinjerto cardíaco; retrasplante cardíaco; trasplante.

Correspondencia:

Dr. Manuel Quiroz.

quirozflores.manuel@gmail.com 


\section{Cardiac Re-transplantation: Case report}

Cardiac transplantation is the best alternative for terminal heart failure, achieving good long-term survival and life quality. One of the most important causes of morbidity and mortality is graft failure, which may be secondary, among others, to acute rejection and / or vasculopathy and its presence requires the consideration of all therapeutic alternatives, re transplantation being one of them.

The results of survival in cardiac retransplantation are good; however, they present the risks of a more intense immunosuppressive therapy as well as the recurrent development of graft vasculopathy. The- refore, it is considered an option in carefully selected patients given that international experience shows that the survival of retransplantation is lower than in primary cases.

We present the case of a 42 year old transplanted patient, who developed graft vascular disease with progressive deterioration of his ventricular function leading to functional class IV. for which a cardiaccardiac retransplantation was performed.

Key words: cardiac allograft failure; heart retransplantation; transplantation.
El trasplante cardíaco (TC) es la terapia de elección en la insuficiencia cardíaca terminal (ICT). La International Society for Heart and Lung Trasplantation (ISHLT) estima que a la fecha se han realizado más de 100.000 TC a nivel mundial. Con los avances en selección del donante, técnicas de preservación cardíaca, evolución en técnica quirúrgica e inmunosupresión, la sobrevida al año es del $86 \%$ y la vida media estimada es cercana a 12 años. ${ }^{1-3}$

Con el incremento del número de pacientes trasplantados, ha aumentado también el número de casos que desarrollan falla del injerto cardíaco, cuyas causas principales corresponden a rechazo agudo y enfermedad vascular del injerto (vasculopatía). Aunque es posible considerar diversas alternativas terapéuticas, cuando se trata de casos refractarios y con deterioro funcional progresivo y significativo, se plantea como opción válida el retrasplante (RT). ${ }^{4-6}$

De acuerdo a los datos de la ISHLT los RT representan el 3\% del total. ${ }^{7-9}$ A continuación comunicamos el primer caso de RT realizado en nuestro hospital.

\section{Presentación del caso}

Paciente de 42 años, de sexo masculino, que el año 2012 inicia cuadro de disnea progresiva de 5 meses de evolución, llegando a capacidad funcional (CF) IV. La ecocardiografía evidenció disfunción sistólica severa con fracción de eyección del ventrículo izquierdo (FEVI) de 15-20\%, hipocinesia difusa, dilatación severa con un trombo apical, insuficiencia mitral moderada a severa, 
ventrículo derecho levemente dilatado e hipertensión pulmonar leve. La coronariografía no demostró lesiones significativas.

El paciente se mantiene con manejo médico por el Comité de Cardiopatía Terminal y Trasplante Cardíaco (CCTyTC). Durante este período evoluciona con episodios de taquicardia ventricular sostenida polimorfa, por lo que se decide implante de cardiodesfibrilador y se ingresa a la lista de espera para TC.

Se realiza TC en mayo del 2013, donante masculino de 44 años, con prueba de reactividad contra el panel de donantes (PRA) de $0 \%$ e inducción con Rituximab. Se realizó TC con técnica bicava y tiempo de isquemia de 124 minutos. Se implantó un marcapasos bicameral previo al alta debido a bloqueo aurículo-ventricular de alto grado.

La biopsia endomiocárdica previa al alta no mostró evidencia de rechazo y se mantuvo en seguimiento clínico, ecocardiográfico, histológico y coronariográfico.

El control ecocardiográfico post operatorio demostró una FEVI del $60 \%$ sin otras alteraciones.
Se manejó con esquema de inmunosupresión con Ciclosporina, Micofenolato y Prednisona. Sin embargo, antes de un año el paciente presentó hipercolesterolemia de predominio LDL, un episodio de gota aguda, dificultad para lograr una presión arterial adecuada y deterioro de la función renal, debido a esto se decide cambio de Ciclosporina por Tacrolimus.

Las biopsias endomiocárdicas realizadas inicialmente cada dos semanas y, posteriormente, cada 3 y 6 meses resultaron negativas para rechazo agudo.

Durante controles ambulatorios se identifica hipertensión arterial de difícil manejo y nuevo deterioro de función renal por lo cual se decide cambiar Tacrolimus por Everolimus el año 2015 Coronariografías para detección de vasculopatía del injerto se realizaron anualmente sin evidenciar alteraciones. Sin embargo, el deterioro de la función renal impedía un seguimiento anual luego del año 2015.

A fines del 2017 presenta dolor precordial intenso, náuseas y vómitos, por lo que es hospitalizado en forma urgente. La ecocardiografía realizada a su ingreso informa

\section{Figura 1.}

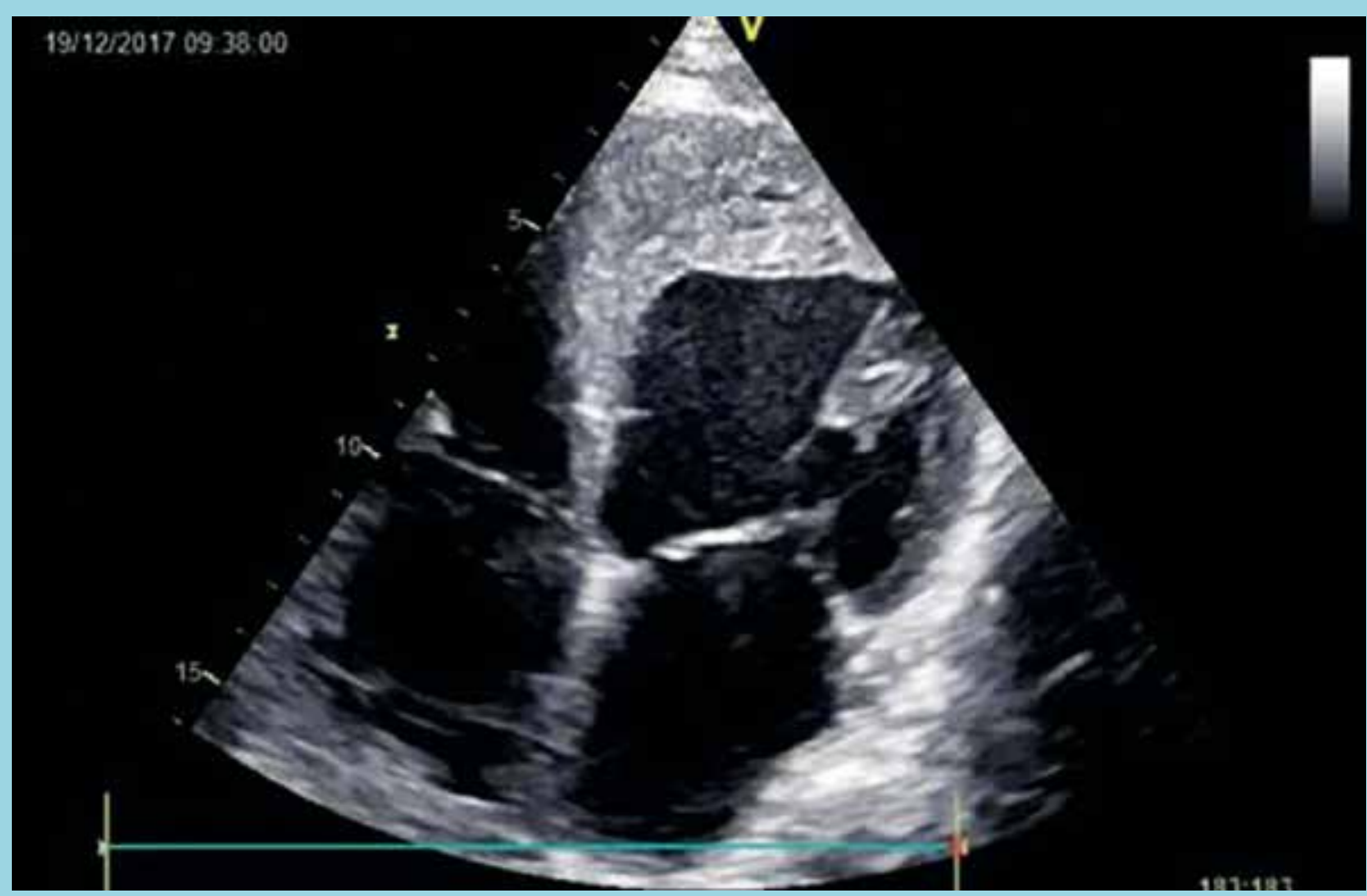

Ecocardiografía control.

Dilatación leve de la aurícula izquierda, acinesia antero septo apical del ventrículo izquierdo con deterioro severo de la función sistólica con fracción de eyección de $31 \%$.

Trombo antero apical, insuficiencia tricuspídea moderada e hipertensión pulmonar leve. 


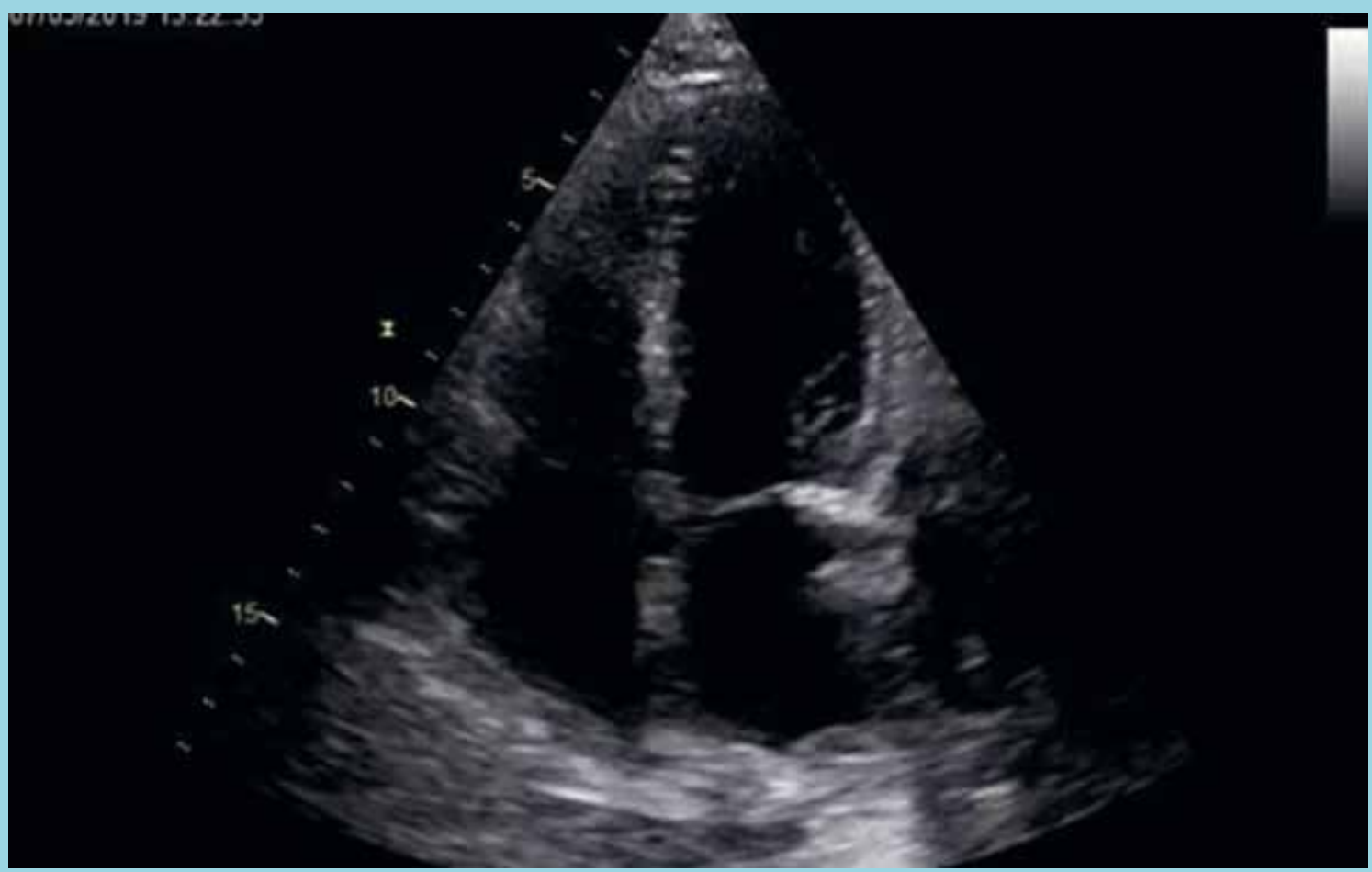

Ecocardiografía de control post trasplante.

Cavidades derechas e izquierda de configuración normal.

Fracción de eyección del ventrículo izquierdo en rango normal.

Leve derrame pericárdico.

FEVI 26\%, cavidades izquierdas dilatadas, extensa acinesia anterior y septal apical, strain longitudinal global $-3.2 \%$ con contraste espontáneo endocavitario compatible con trombo intraventricular. Además, presentaba disfunción diastólica tipo III, insuficiencia mitral y tricuspídea leve, presión sistólica de arteria pulmonar de $55 \mathrm{mmHg}$. (Figura 1).

El control de biomarcadores de daño miocárdico detectó CK-MB 259 y Troponina ultrasensible 29.205. Se practica una coronariografía inmediata que mostró oclusión proximal de arteria descendente anterior (DA) de aspecto agudo y lesión de rama de arteria circunfleja (primera obtusa marginal). Se realiza aspiración de trombos y se instala stent medicado en DA y en obtusa marginal en forma diferida sin incidentes. Su curso posterior es estable y se indica alta (Figura 2).

El paciente presenta nuevo episodio de insuficiencia cardíaca global, por lo que se hospitaliza y se realiza manejo médico en unidad de cuidados intensivos hasta lograr compensación clínica.

Durante el año 2018 se evidencia evolución clínica con insuficiencia cardíaca, asociado a episodios de falla renal aguda. El estudio nefrológico demuestra riñones de estructura normal a la ecotomografía y mínima proteinuria de 24 horas. El sondeo derecho mostró resistencia vascular pulmonar de 2.2 unidades Wood; valor de pro BNP mayor a 2.000 y creatinina elevada. Con estos antecedentes, el CCTyTC define que es candidato para RT $\mathrm{y}$ es inscrito en lista de espera.

El 28 de enero del 2019 se realiza TC, con donante sexo femenino de 22 años, APR 0\%, inducción con Rituximab. Se constata extenso proceso adherencial y un corazón severamente dilatado, teniendo un tiempo de isquemia de 125 minutos (Figura 3).

Ingresa a unidad de cuidados intensivos con apoyo de drogas vasoactivas, diuresis conservada pero con evidencia de sangrado persistente, por lo que es sometido a reintervención para aseo y hemostasia de hemorragia difusa. Evoluciona estable en su hemodinamia y es extubado a las 24 horas, sin embargo, presenta oligoanuria que requiere de hemodiálisis, recuperando función renal al cabo de 5 días. Al séptimo día se encuentra sin re- 


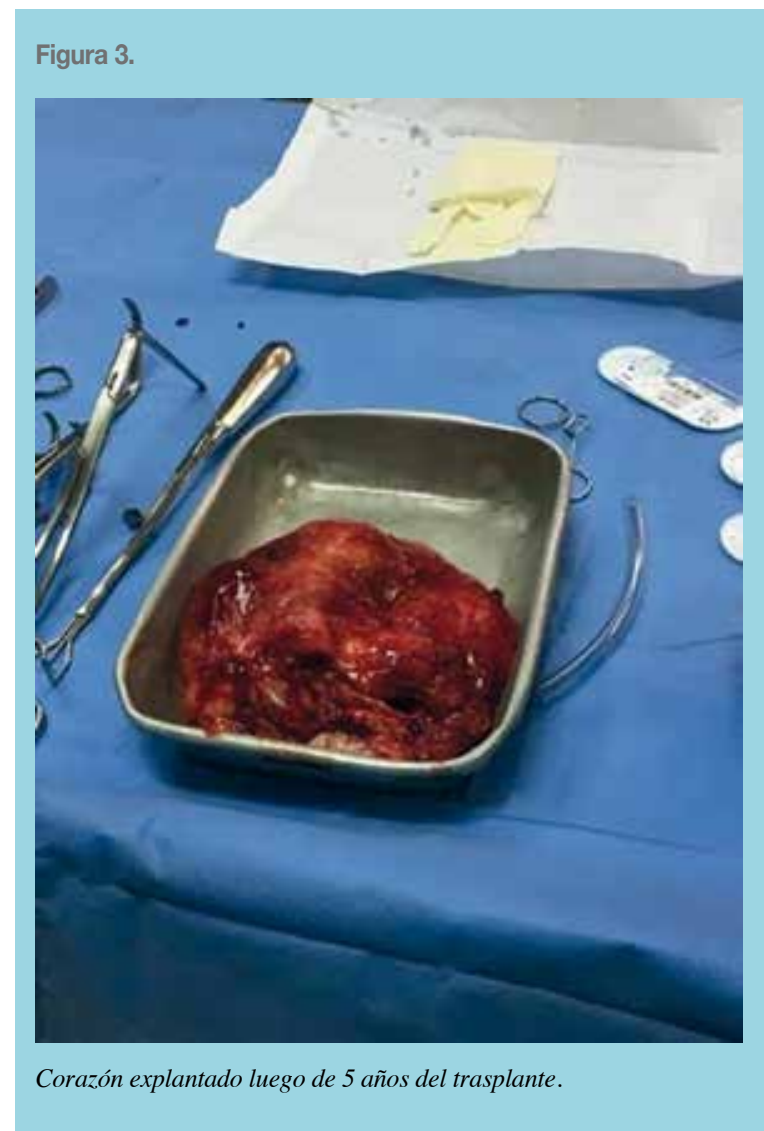

querimiento de apoyo vasoactivo y es trasladado a unidad de cuidados intermedios. El 7 de febrero se realiza biopsia endomiocárdica y el control ecocardiográfico es informado con parámetros en rangos normales, siendo dado de alta el 12 de febrero, a quince días de realizado el RT (Figura 4).

Ha sido controlado en forma ambulatoria, iniciado rehabilitación cardiovascular y se ha mantenido en buenas condiciones generales (CF I).

\section{Discusión:}

Durante los últimos 20 años, la edad de los pacientes trasplantados ha aumentado de forma sostenida y las edades más extremas también han aumentado.

La miocardiopatía y la enfermedad coronaria son las principales causas de insuficiencia cardíaca que lleva a trasplante, con un aumento progresivo de las miocardiopatías y, a su vez, con pacientes con más comorbilidad como destaca el registro de la International Society for Heart and Lung trasplantation (ISHLT) en que se observa que se realizan trasplantes en pacientes cada vez más complejos, con alta prevalencia de comorbilidades como diabetes $(25 \%)$, hipertensión arterial (46\%), en-

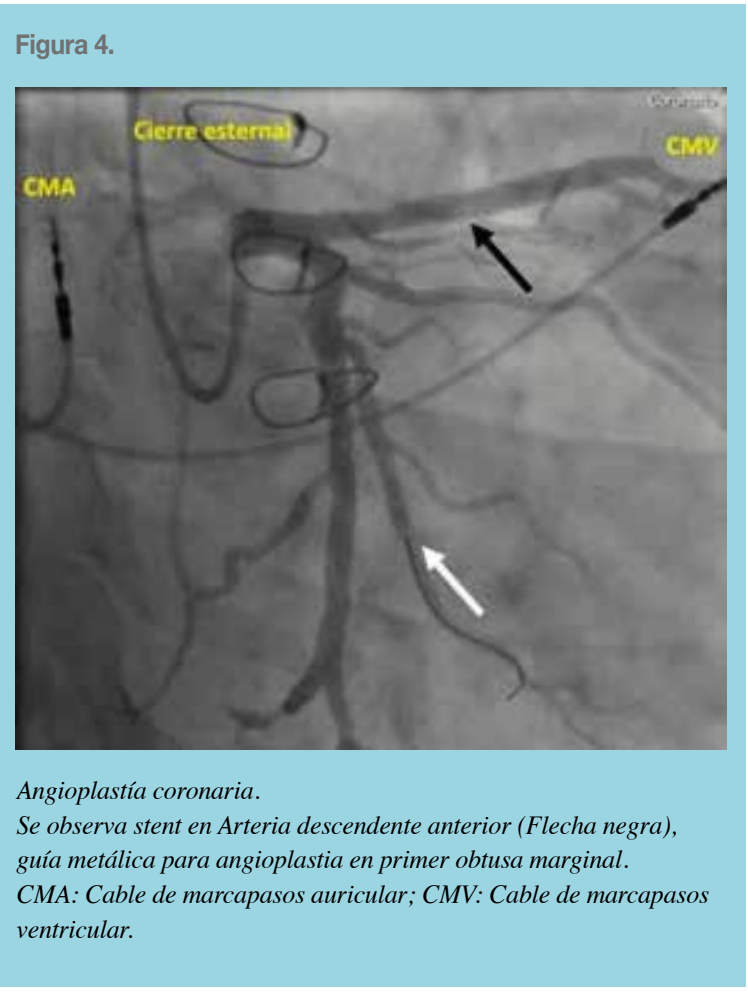

fermedad pulmonar obstructiva crónica $(4 \%)$, historia de insuficiencia renal (4\%), enfermedad neoplásica previa $(7 \%)$ y cirugía cardíaca previa $(47 \%){ }^{10-13}$

El porcentaje de RT cardíaco se ha mantenido estable desde 1982 encontrándose entre un 2 y un 4\%; durante el período 2006 a 2013, de un total de 26.294 trasplantes, los RT correspondieron al 3.1\%.12,13 Sin embargo, los datos nacionales hablan de una casuística de RT mucho menor y en nuestro centro este es el primer caso realizado.

El riesgo de RT aumenta a medida que avanzan los años desde el trasplante y ocurre, principalmente, después de 10 años, siendo más común en el grupo etario entre 18 a 39 años, alcanzando un $5 \%$ en este grupo. ${ }^{10,11}$

La vasculopatía del injerto (VI) y la miocardiopatía son las principales causas de RT, y la VI presenta una mayor frecuencia en Norte América. La falla primaria del injerto y el rechazo corresponden a un $25 \%$ de las causas de RT en Europa y Norte América. La falla primaria del injerto corresponde a la principal causa de RT durante el primer mes del trasplante y la VI es la principal indicación posterior a un año del trasplante. 11-13

En nuestro caso el estudio de biopsias endomiocárdico no mostró signos de rechazo agudo, y el seguimiento con coronariografía anual se vio limitado al tercer año posterior al TC, debido a los episodios constantes de insuficiencia renal aguda. Sin embargo, en el estudio 
publicado por Torres y cols (2011), destaca la baja capacidad de la coronariografía para identificar vasculopatía del injerto durante los primeros años, debido a que el daño es, principalmente, difuso y probablemente no logramos identificarlo de forma precoz.

Nuestro caso es un paciente joven que cursó con episodios de hospitalización frecuentes por insuficiencia cardíaca e insuficiencia renal aguda, con necesidad de ventilación mecánica y uso de drogas inotrópicas lo que es compatible con lo reportado en pacientes que requieren un $\mathrm{RT}^{12}$

La sobrevida del trasplante es cercana al $85 \%$ a un año y $69 \%$ a 5 años, mientras que la sobrevida posterior a un RT es inferior, llegando al $70 \%$ al año y $38 \%$ a 10 años. Respecto a esto, el factor más importante es la causa que llevó al trasplante primario.9-13

La VI es la causa de RT con mejor pronóstico y mejor sobrevida al primer año, mientras que los pacientes sometidos a RT por fallo primario del injerto presentan mortalidad a un año de $46 \% .^{11}$

Las causas de muerte más comunes en pacientes trasplantados y RT son la falla del injerto, infecciones y la falla multiorgánica, especialmente, durante los primeros años. Por otro lado, las neoplasias malignas, la VI y la insuficiencia renal se hacen más prevalentes a partir del tercer año del trasplante.

A su vez, las causas de muerte presentan diferencias entre TP y RT, en el que es más común la falla del injerto y la falla multi-orgánica. ${ }^{11,12}$

Las drogas de inducción en inmuno supresión son similares en trasplante y en RT con el uso de globulinas policlonales anti-linfocito y globulinas policlonales anti-timocito conjunto con antagonistas del receptor de interleukina ${ }^{2}$.

La inmuno supresión de mantención se basa principalmente en el tacrolimus como principal inhibidor de la calcineurina; el micofenolato mofetil y el ácido micofenólico son los inhibidores del ciclo celular de elección. ${ }^{9,11,12}$

El uso de corticoides desciende luego de los 5 años, cambiando casi en la totalidad de los casos de TP por las terapias inhibitoras de la proteína mTOR. ${ }^{10,11}$

El rechazo es más común después de un RT, sin embargo, esto no es estadísticamente significativo y las terapias inmuno supresoras han disminuido esta reacción en los TP y en los RT. La incidencia de rechazo agudo disminuyó desde el período 2004 - 2006 de un $30 \%$ para TP y un $36 \%$ para RT a un $25 \%$ y $27 \%$, respectivamente, durante el período 2010 a 2011 . Además, hoy en día se conoce que no es necesario tratar de forma aguda el rechazo celular moderado, con lo que el tratamiento para el rechazo agudo disminuyó tanto para los TP como los RT.

En relación a la terapia actual, el uso de estatinas, antiplaquetarios y micofenolatos ha disminuido la incidencia de vasculopatía del injerto. Sin embargo, cuando el paciente presenta insuficiencia cardíaca terminal la alternativa terapéutica de elección es el RT, teniendo en cuenta la menor sobrevida y la recurrencia de las alteraciones que llevaron a la falla del injerto.

\section{Agradecimientos:}

Enfermera Paulina Espinoza Reed, programa de procura y trasplante cardíaco del Hospital Dr. Gustavo Fricke. Equipo de la Unidad de Paciente Crítico Cardiovascular del Hospital Dr. Gustavo Fricke. 


\section{Referencias}

1. HUNT SA, HADDAD F. The changing face of heart transplantation. J Am Coll Cardiol 2008; 52: 587-98.

2. LUND LH, EDWARDS LB, KUCHERYAVAYA AY, et al. The Registry of the International Society for Heart and Lung Transplantation: Thirtieth Official Adult Heart Transplant Report--2013; focus theme: age. J Heart Lung Transplant 2013; 32: $951-64$

3. TAYLOR DO, EDWARDS LB, BOUCEK MM, et al. Registry of the International Society for Heart and Lung Transplantation: twenty-fourth official adult heart transplant report--2007. J Heart Lung Transplant 2007; 26: 769-81.

4. DZEMESHKEVICH S, RAGIMOV A, MIKHAYLOV Y, et al. Plasmapheresis in the treatment of posttransplant cardiomyopathy. Artif Organs 1998; 22: 197-202.

5. BENZA RL, ZOGHBI GJ, TALLAJ J, et al. Palliation of allograft vasculopathy with transluminal angioplasty: a decade of experience. J Am Coll Cardiol 2004; 43: 1973-81.

6. HALLE AA 3RD, DISCIASCIO G, MASSIN EK, et al. Coronary angioplasty, atherectomy and bypass surgery in cardiac transplant recipients. J Am Coll Cardiol 1995; 26: 120-8.

7. MEHRA MR. Contemporary concepts in prevention and treatment of cardiac allograft vasculopathy. Am J Transplant 2006; 6: 1248-56.

8. PHAN K, LUC JGY, XU J, et al. Utilization and Outcomes of Temporary Mechanical Circulatory Support for Graft Dysfunc- tion After Heart Transplantation. ASAIO J 2017; 63: 695-703.

9. LUND LH, KHUSH KK, CHERIKH WS, et al. The Registry of the International Society for Heart and Lung Transplantation: Thirty-fourth Adult Heart Transplantation Report-2017; Focus Theme: Allograft ischemic time. J Heart Lung Transplant 2017; 36: 1037-46.

10. STEHLIK J, EDWARDS LB, KUCHERYAVAYA AY, et al. The Registry of the International Society for Heart and Lung Transplantation: 29th official adult heart transplant report-2012. J Heart Lung Transplant 2012; 31: 1052-1064.

11. GOLDRAICH LA, STEHLIK J, KUCHERYAVAYA A Y, EDWARDS L B, ROSS H J. Retransplant and Medical Therapy for Cardiac Allograft Vasculopathy: International Society for Heart and Lung Transplantation Registry Analysis. American Journal of Transplantation 2016; 16: 301-309.

12. LUND L.H., EDWARDS L.B., KUCHERYAVAYA A.Y., BENDEN C., CHRISTIE J.D., Dipchand A.I., Dobbels F., The registry of the international society for heart and lung transplantation: Thirty-first official adult heart transplant report - 2014; Focus theme: Retransplantation. J Heart Lung Transplant 2014; 33: 996-8.

13. K. KHUSH K., CHERIKH W.S., CHAMBERS D.C., GOLDFARB S., HAYES D., KUCHERYAVAYA A.Y., LEVVEY B.J. The International Thoracic Organ Transplant Registry of the International Society for Heart and Lung Transplantation: Thirty-fifth Adult Heart Transplantation Report-2018; Focus Theme: Multiorgan Transplantation, J Heart Lung Transplant 2018; 37: 1155-68. 